\title{
INERTIAL SENSOR BASED POST FALL ANALYSIS FOR FALSE ALARMING REDUCTION
}

\author{
Lin $\mathrm{Ye}^{1}$, Kai Cao ${ }^{1}$, Jay Guo ${ }^{1}$, Xiaojing Huang ${ }^{1}$, Peter Beadle ${ }^{2}$, Ahmadreza Argha ${ }^{1}$, Massimo Piccardi ${ }^{1}$, Guangquan Zhang ${ }^{1}$, \\ and Steven W. Su${ }^{1}$ \\ ${ }^{1}$ Faculty of Engineering and Information Technology \\ University of Technology, Sydney \\ NSW 2007 Australia \\ Email: \{Lin.Ye-1, Kai.Cao-1\}@student.uts.edu.au, \{Jay.Guo, \\ Xiaojing.Huang,Ahmadreza.Argha, Massimo.Piccardi, Guangquan.Zhang, \\ Steven.Su\}@uts.edu.au \\ ${ }^{2}$ LoneAlarm Pty Ltd \\ 38-46 South Street, Rydalmere NSW 2116 Australia \\ Email: peter@lonealarm.com
}

\begin{abstract}
One of the major public health problems among elderly people is falling injury. This study investigates fall detection and prevention by using inertial sensors for which the major existing challenging is how to significantly reduce false alarming in order to enhance the acceptance of elderly users during rehabilitation and daily exercises. Different from most existing approaches in the literature, the behavior after falling will be analyzed in details, which can not only greatly reduce false alarming, but also significantly improves the accuracy of the assessment of the severity of falling injuries.
\end{abstract}

\section{KEY WORDS}

Fall detection, Inertial sensor, Post fall behavior.

\section{Introduction}

In light of the World Health Organization, falls are recognized as a major cause of injury for elder people. Without preventative measures, the estimated fall-related injuries will increase $100 \%$ in 2030 [1]. Hence, fall detection has been actively investigated in the past few years.

The fall detection can be divided into two major categories, the wearable motion-record sensor (e.g., accelerometer, gyroscope, and so on) based systems and the contextaware systems [2]. Comparing to the context-aware systems, the wearable motion-record sensor based systems are both convenient and flexible. Especially, nowadays, due to the rapid development of MEMS technology, the motionrecord sensors are becoming lightweight, small and low power consumption, and can be easily inserted to clothes, gloves, shoes and so on, to form wearable monitoring systems. Furthermore, newly-launched smartphones come with a rich set of embedded sensors, such as an accelerometer, digital compass, gyroscope, GPS, microphone, and camera [3]. Several researches are currently taking advan- tage of embedded sensors to develop smartphone-based fall detectors [2].

For wearable sensor-based fall detection systems, the major technique in the previous researches can be summarized as single-feature threshold-based methods $[4,5,6]$ and multiple-feature machine learning based methods $[7,8$, 9]. Although the machine learning based methods are more complicated, it can often achieve higher detection accuracy compared to the threshold-based methods. The rapid growth of the Internet of Thing (IoT) and cloud computing is driving the multiple-feature machine/statistical learning based approaches to become prevalent. Nevertheless, most studies conducted on these two kinds of methods were mainly focused on falling detection alone without evaluating the severity of the fall. Moreover, only a few investigations investigate the fall detection in free-living condition, i.e., the study of distinguishing fall from the activities of daily living (ADL) is insufficient [2]. Therefore, most existing fall detection methods suffer from high false alarm rate in real life situation [10]. Hence, it is necessary to improve the utility of existing methods in free-living conditions.

Recently, Schwickert [11] explored post fall analysis by investigating the movement patterns of getting up from floor based on standalone IMUs. Several features have been proposed and applied to distinguish between critical incidents and self-recovered falls. Schwickert has shown these features can provide valuable information for the discrimination of falling from ADL. However, the research in [11] is based on expensive high-performance standalone IMU (APDM), and it also requires the user bonding these IMUs to some specific positions of their body. At present, smartphones are widely used and equipped with integrated IMU which can provide rich information such as acceleration, angular velocity and orientation information (i.e., roll, yaw, and pitch). In this study, we investigate the feasibility of utilizing smartphone alone and compare the results with 
high-performance standalone IMU for post fall analysis. It should be pointed out that the IMU integrated into smartphones may not achieve the same performance of high end IMU, due to various limitations, e.g., size and data sampling/transferring rate limitations. To this end, in this study, we will propose different features based on the IMU embedded in a smartphone.

In real life situation, people often put their smartphone in the pocket during daily life or place it on the upper arm during exercise. Under this consideration, this study focused on the movement features extracted from smartphones located in the pocket and/or bonded to the upper arm. Furthermore, it is worth pointing out that the degree of gait deviation before and after fall will be highly related to the severity of fall. Motivated by this fact, this study provided an index to measure the degree of the gait asymmetry to assess the severity of falls. Due to gait differences between individuals, the movement pattern of getting up may vary greatly among different subjects. For this reason, this research aimed to provide an individualized gait analysis by analyzing the historical data of each individual user based on clouds computing to individually identify the degree of gait deviations after fall. This is able to not only evaluate the severity of fall but also greatly reduce the false alarm rate to enhance the acceptance of wearable health monitoring systems.

Actually, post fall analysis have been clinically requested in Australia. In the documents from Queensland Health website [12] regarding Falls Injury Prevention Model Implementation Standard, it is stated that post falling analysis shall be conducted following a fall which generates an interdisciplinary care plan to reduce the risk of future falls and address any identified co-morbidities or falls risks factors. The level of analysis should reflect the seriousness resulting consequences to the patient. An indepth analysis is required if serious injury or death has resulted from a fall. In this study, the smartphone-based post-fall analysis will potentially provide a firm support for clinical post-fall analysis by using big data analysis based on the fact of the growing prevalence of smartphones in the daily lives.

This paper is organized as follows. In Section 2, equipment and data pre-processing are introduced. In Section 3, the methodology of feature selection is analyzed and symmetric assessment of walking is proposed. In Section 4, experimental results are discussed. Section 5 concludes the paper.

\section{Equipment and Data Pre-processing}

\subsection{Equipment}

In this study, an iPhone 6s was selected for data collection. Same as other types of smartphones, e.g., Androidbased smartphones, iPhone has several built-in motionrecording sensors, such as an accelerometer, gyroscope, magnetometer, and so on. The iPhone $6 \mathrm{~s}$ is able to pro- vide orientation information, gravity data, and linear acceleration to developers directly, which is calculated by an embedded motion microprocessor using raw acceleration, angular velocity and magnetic field measurements. These processed data is accessible to the developers and the users with relatively high accuracy. The developers and researchers of the earlier version smartphone often have to use Kalman filter based data fusion techniques to estimate the orientation information from raw measurement data externally [13]. However, due to the limitation of the output frequency of accelerometer and gyroscope of smartphone (around 100Hz), the Kalman filter based external estimation can hardly achieve accurate estimation [14]. On the contrary, with the power of internal motion processor, the internal orientation updating rate is much higher than $100 \mathrm{~Hz}$ although the output frequency of orientation data is still around $100 \mathrm{~Hz}$. Thus, relatively accurate orientation information can be provided in newly-launched smartphones. However, it should be noted that the velocity and distance data from IMU of iPhone 6s were not as accurate as expected based on our experiments. Especially the distance, which is calculated by double integrating acceleration signal, often has large estimation error.

For symmetric analysis, in this study, we selected two commercial-grade IMU modules for both ankles. One of the IMU module used in this study is manufactured by ImeasureU, which contains one triaxial accelerometer sensor, one triaxial gyroscope sensor, and one triaxial magnetometer sensor. The accelerometers are set to $\pm 16 \mathrm{~g}$ measurement range with 16 bits resolution, gyros are set to $\pm 2000^{\circ} / \mathrm{s}$ sensitivity range with 16 bits resolution, and magnetometers to $\pm 1200 u T$ with 13 bits resolution respectively. The module supports Bluetooth communication and it can log 9-axis raw data at $500 \mathrm{~Hz}$ on internal SD card. Later on, the logged data can be downloaded via micro USB to the computer. Alternatively, the IMU module can also wireless steam 9 axis data to smartphone in real time at $100 \mathrm{~Hz}$.

Furthermore, we also included two SensorTags in the experiment for symmetric analysis. The SensorTag is developed by Texas Instrument (TI) as a developer platform of IoT. SensorTag contains an IMU MPU9250 which is widely used in smartphone and health bracelet. SensorTag supports cloud service which is important for IoT. In this experiment, two SensorTags are placed on the medial of the ankles.

The IMU modules from ImeasureU, SensorTag and iPhones are placed in the positions as shown in Fig (1).

\subsection{Data Pre-processing}

Due to the bias variation and parameter instability of MEMS sensors, calibration is necessary in order to obtain accurate measurements. In this research, the accelerometer and gyroscope from iPhone, ImeasureU IMU module and SensorTag were individually calibrated before experiments to enhance the reliability of measurement. 


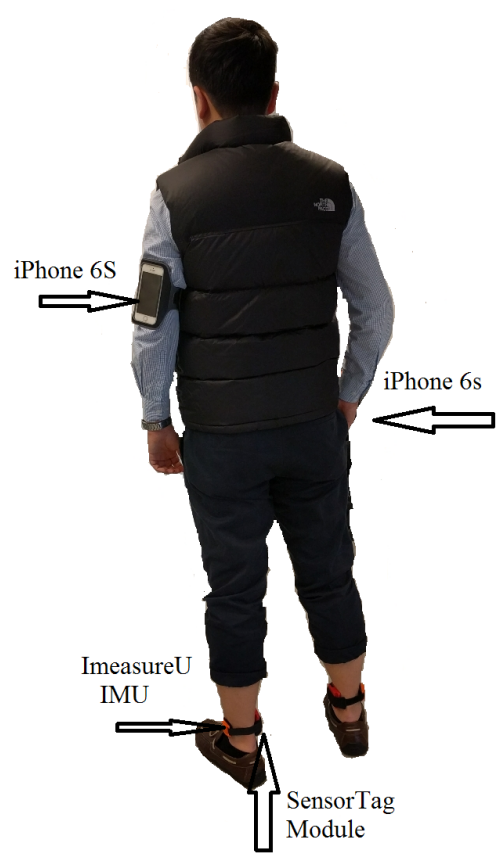

Figure 1. The positions of the smartphone, ImeasureU IMU module and SensorTag during experiments

\subsubsection{Accelerometer Calibration}

For accelerometer, we selected a 6-parameter model which can provide similar accuracy with 9-parameter model [15]. The mathematical model between local acceleration $\mathbf{A}=$ $\left[a_{x}, a_{y}, a_{z}\right]^{T}$ and measured value $\mathbf{V}=\left[v_{x}, v_{y}, v_{z}\right]^{T}$ can be expressed as:

$$
\left[\begin{array}{l}
a_{x} \\
a_{y} \\
a_{z}
\end{array}\right]=\left[\begin{array}{ccc}
S_{x} & 0 & 0 \\
0 & S_{y} & 0 \\
0 & 0 & S_{z}
\end{array}\right]\left(\left[\begin{array}{l}
v_{x} \\
v_{y} \\
v_{z}
\end{array}\right]+\left[\begin{array}{l}
o_{x} \\
o_{y} \\
o_{z}
\end{array}\right]\right),
$$

where the orthogonal matrix $\mathbf{S}=\left[\begin{array}{llllllll}S_{x} & 0 & 0 ; 0 & S_{y} & 0 ; & 0 & 0 & S_{z}\end{array}\right]$ is associated with scale factor and $\mathbf{O}^{\mathbf{T}}=\left[o_{x}, o_{y}, o_{z}\right]$ is related to the offset.

The method from [16] is applied for accelerometer calibration, which is an optimal calibration method in terms of optimal experimental design. However, it should be clarified, when using the internally processed linear acceleration signal provided by iPhone $6 \mathrm{~s}$, we directly use it without calibration.

\subsubsection{Gyroscope Calibration}

MEMS gyroscope is suffering from drift problem in real life situation, especially for long-term measurement. However, in this application, the measurements of getting up are usually sampled within 30s. Therefore, a simple calibration to remove static drift should be sufficient. Due to the fact that the output of gyroscope should be zero in the static state, we can simply take the mean value of static

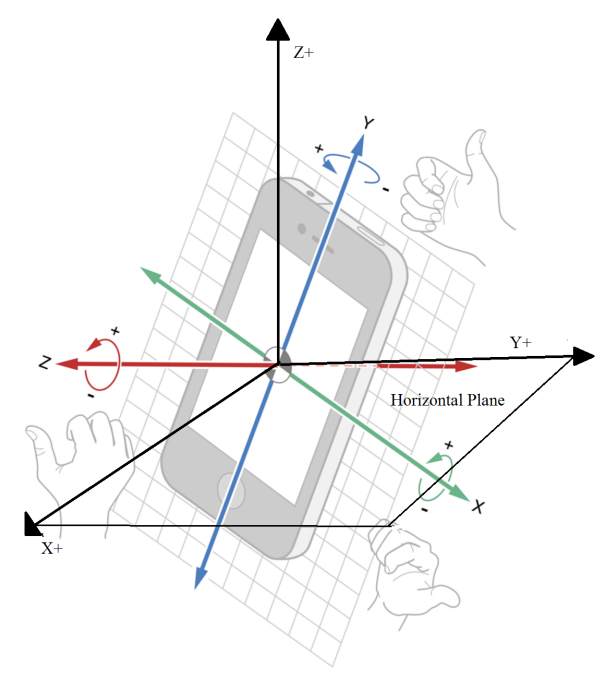

Figure 2. iPhone's coordinate system and its reference world coordinate system

state measurement for $30 \mathrm{~s}$ as the drift and then compensate it in the measurement accordingly.

\subsubsection{Acceleration and Angular Velocity}

With calibrated accelerometer and gyroscope, we can obtain relatively accurate acceleration and angular velocity respectively. As we mentioned above, the orientation information between the smartphone and the human body is unknown. It is necessary to transfer the acceleration and angular velocity from body-fixed coordinate system to world coordinate system. For iPhone 6s, its output contains orientation information including quaternions and Euler Angles, so acceleration and angular velocity from body-fixed coordinate can be transferred to world coordinate system [17].

Fig.(2) shows the relationship between iPhone's coordinate system and its reference world coordinate system.

With this reference coordinate system and orientation data, the linear acceleration and angular velocity on the reference world coordinate system can be calculated. With roll $(\phi)$, pitch $(\theta)$ and yaw $(\psi)$, the relationship between the body-fixed coordinate system and the reference world coordinate system can be expressed as:

$$
\left[\begin{array}{c}
a_{x, \text { world }} \\
a_{y, \text { world }} \\
a_{z, \text { world }}
\end{array}\right]=C_{\text {world }}^{\text {body }}\left[\begin{array}{l}
a_{x, \text { body }} \\
a_{y, \text { body }} \\
a_{z, \text { body }}
\end{array}\right]
$$

where $C_{\text {world }}^{\text {body }}$ is the coordinate transformation matrix from body-fixed coordinate to reference world coordinate. It is 
composed from the following three rotation matrices:

$\left[\begin{array}{ccc}c(\psi) & -s(\psi) & 0 \\ s(\psi) & c(\psi) & 0 \\ 0 & 0 & 1\end{array}\right]\left[\begin{array}{ccc}c(\theta) & 0 & s(\theta) \\ 0 & 1 & 0 \\ -s(\theta) & 0 & c(\theta)\end{array}\right]\left[\begin{array}{ccc}1 & 0 & 0 \\ 0 & c(\phi) & -s(\phi) \\ 0 & s(\phi) & c(\theta)\end{array}\right]$,

where $c$ represents $\cos$ and $s$ represents $\sin$.

For ImeasureU IMU module and SensorTag, as we used it for symmetric analysis only, it is unnecessary to transfer it to the world coordinate.

\subsubsection{Data Filtering}

The data from iPhone has been filtered already by internal motion processor, and it is unnecessary for extra filtering works. For the data from IMU modules, discrete wavelet transform (DWT) has been employed for the purpose of noise reduction. The DWT computes successive convolutions between input signal with the discrete low pass and high pass filters [18]. The output frequency from the IMU module is $500 \mathrm{~Hz}$, with third order DWT, the remained signal from low pass filter still has $62.5 \mathrm{~Hz}$ which is sufficient for body movement measurement.

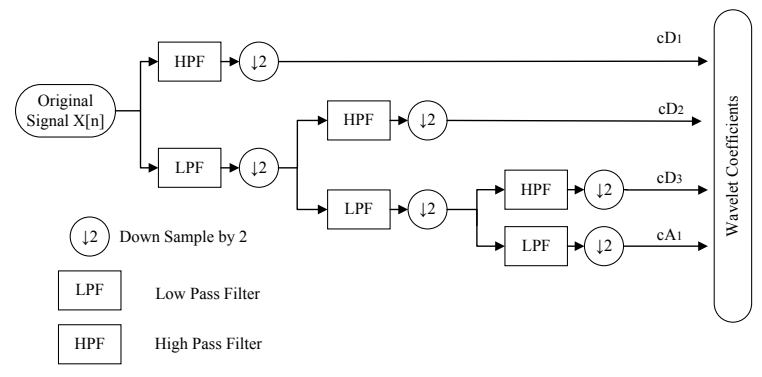

Figure 3. The application of discrete wavelet transform

\section{Methodologies}

This research is carried out to consummate previous fall detection algorithm [7]. Fig.(4) shows the schematic diagram of this method. Whenever a fall is detected, the post fall analysis is then activated to exam the features of getting up. With baseline information perhaps recorded in a remote server, this method can help to reduce the false alarm caused by ADL. Meanwhile, the values of the extracted features can be consulted to evaluate the severity of the fall. Moreover, the symmetric analysis will be carried out to further remove the false alarm, which can also reduce the risk of true negative. With this method, only for those assessed as critical falls, this fall detection system will inform the user and send help information remotely to a local medical center.

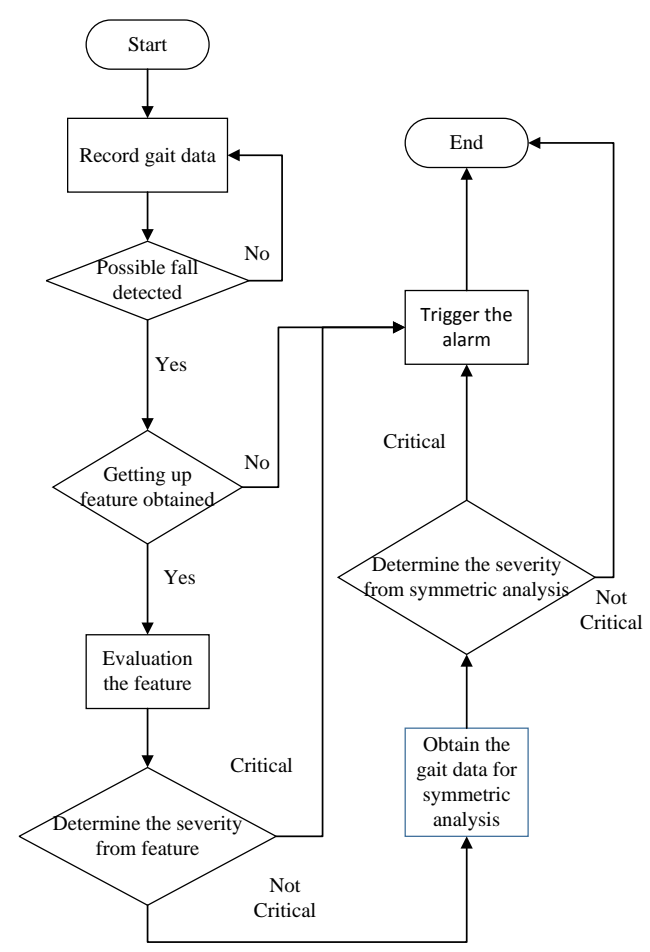

Figure 4. Schematic diagram

\subsection{Feature}

Several features are selected for the experiments, some of these features are coming from previous research which have been applied in high-performance standalone IMU. We will apply these features with smartphone intergraded IMU to find out the feasibility and validity of these features. Furthermore, some new features from [19] will also be tested for the assessment of movement patterns of getting up after fall.

- Transfer Duration. Given a time series of acceleration signal from the accelerometer, the start point $\left(T_{\text {start }}\right)$ is detected when the magnitude of acceleration reach a standard deviation threshold $\left(\sigma_{a_{\text {norm }}}\right)$. For the end point $\left(T_{\text {end }}\right)$ of the signal, it is detected by the same threshold for start point. Furthermore, to increase the robustness of the detection, it is necessary to include a standard deviation threshold from the angular velocity $\left(\sigma_{\omega_{n o r m}}\right)$. The transfer duration $\left(T_{t d}\right)$ can be calculated by:

$$
\begin{gathered}
T_{\text {start }}=t, \sigma_{a_{\text {norm }}}(t)>0.05 \mathrm{~m} / \mathrm{s}^{2} \wedge \sigma_{\omega_{\text {norm }}}>0.1 \mathrm{rad} / \mathrm{s}, \\
T_{\text {end }}=t, \sigma_{a_{\text {norm }}}(t)<0.05 \mathrm{~m} / \mathrm{s}^{2} \wedge \sigma_{\omega_{\text {norm }}}<0.1 \mathrm{rad} / \mathrm{s} \\
T_{t d}=T_{\text {end }}-T_{\text {start }} .
\end{gathered}
$$

Generally, the transfer duration is used to indicate the time duration of people standing up after fall. It is 
obvious that people will have longer transfer duration after critical fall or several unsuccessful attempting of getting up.

- Transfer Angular Velocity. Transfer angular velocity is an index of root mean square of the rotational speed $\left(\omega_{i=x, y, z}\right)$, which is calculated separately based on the three axes of the gyroscope. It is applied to describe the angular movement during transfer:

$$
\begin{aligned}
& R M S \omega_{x}=\sqrt{\frac{\sum_{t=T_{\text {start }}}^{T_{\text {end }}} \omega_{x}(t)^{2}}{f_{s} T_{t d}}}, \\
& R M S \omega_{y}=\sqrt{\frac{\sum_{t=T_{\text {start }}}^{T_{\text {end }}} \omega_{y}(t)^{2}}{f_{s} T_{t d}}}, \\
& R M S \omega_{z}=\sqrt{\frac{\sum_{t=T_{\text {start }}}^{T_{\text {end }}} \omega_{z}(t)^{2}}{f_{s} T_{t d}}} .
\end{aligned}
$$

where $f_{s}$ is the sampling frequency.

- Jerk-Smoothness. Jerk $\left(J_{i=x, y, z}\right)$ is a sensor-based measurement feature. It is defined as the change of the acceleration signals during the transfer duration which is applied to quantify smoothness and coordination. It is calculated separately for each axis. Based on Jerk, smoothness $\left(S M_{i=x, y, z}\right)$ can explain whether the movement sequence is toward a clear direction continuously and linearly or not. It is one of the Jerkbased Measures introduced by Schwickert, and the smoothness is the sum of the jerk and normalized by the transfer duration:

$$
\begin{aligned}
J_{x} & =f_{s}\left(a_{x, k}-a_{x, k-1}\right), \\
J_{y} & =f_{s}\left(a_{y, k}-a_{y, k-1}\right), \\
J_{z} & =f_{s}\left(a_{z, k}-a_{z, k-1}\right), \\
S M_{x} & =T_{T_{t d}}^{3} \sum_{t=T_{\text {Start }}}^{T_{\text {end }}}\left|J_{x}(t)\right|, \\
S M_{y} & =T_{T_{t d}}^{3} \sum_{t=T_{\text {Start }}}^{T_{\text {end }}}\left|J_{y}(t)\right|, \\
S M_{z} & =T_{T_{t d}}^{3} \sum_{t=T_{\text {Start }}}^{T_{\text {end }}}\left|J_{z}(t)\right| .
\end{aligned}
$$

where $f_{s}$ is the sampling frequency.

- Willison Amplitude. Willison Amplitude is defined as the number of amplitude change when the difference between two consecutive samples exceeds a threshold $\varepsilon$,

$$
W A=\sum_{t=1}^{T-1} h(t)
$$

where

$$
h(t)=\left\{\begin{array}{l}
1, \text { if }|v(t)-v(t-1)|>\varepsilon \\
0, \text { otherwise }
\end{array}\right.
$$

For the testing of WA feature, we will take angular velocity from three axes as $v(t)$ showed in (17).

- Slope Sign Change. Slope sign change is applied to count the number of times when the sign of the slope alters among three continuous samples. A threshold $\varepsilon_{2}$ is necessary to ensure the difference is significant instead of causing by noise.

$$
\begin{gathered}
S S C=\sum_{t=1}^{T-1} g(t), \\
h(t)= \begin{cases}1, & \text { if }|v(t)-v(t-1)| \cdot \\
|v(t)-v(t+1)|>\varepsilon_{2} \\
0, & \text { otherwise. }\end{cases}
\end{gathered}
$$

We will take acceleration and angular velocity from three axes as $v(t)$ to test this feature.

\subsection{Symmetric analysis}

Extensive researches about gait analysis have been done with IMU module [20][21]. For this study, the IMUs are placed on both ankles as shown in Fig.(5). We select the angular velocity around $\mathrm{z}$ axis as it can effectively describe footsteps when comparing to the other two axes [20].

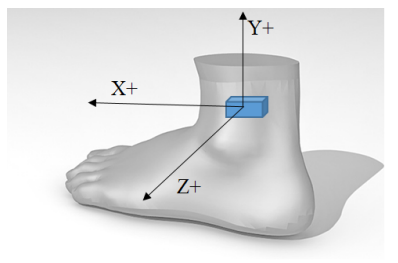

Figure 5. IMU position and orientation on ankle

For this post-fall analysis, we are focusing on the change of gait symmetry. Hypothetically, for selfrecovered fall, the change of gait before and after fall should be minor while critical fall can cause a significant change in gait. Fig.(6) shows the gyroscope data recorded from a subject with IMU mounted on his/her right ankle. During the experiments, a heavy sandbag has been tied up in the subject's leg to simulate serious injury. In Fig.(6), the top one is the data from normal walking, while the bottom one shows the data of walking with the heavy sandbag. It can be observed that the frequency and magnitude are significantly different.

To quantify the difference, we select one integrity period between two peaks. We use $P_{n}$ and $P_{i n j}$ to represent these two selected periods from normal walking and walking with sandbag respectively. We applied the method from [22] to align the signal, then the different can be expressed as: 

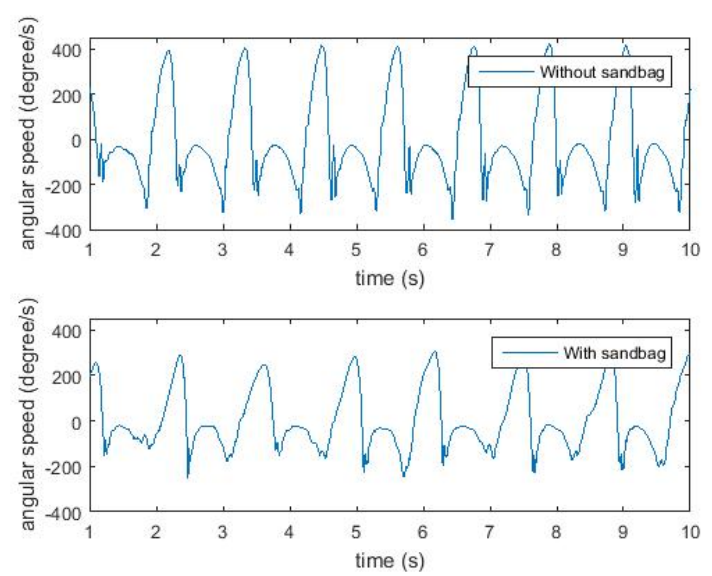

Figure 6. Gait information for walking under different conditions

$$
\mathcal{I}_{\text {diff }}=\frac{1}{N} \sum_{t=1}^{N}\left(P_{n}(t)-P_{\text {inj }}(t)\right) / P_{n}(t),
$$

where $N$ is the mutual length of two selected samples.

\section{Experimental Results and Discussions}

\subsection{Experiments}

The experiments for this research were undertaken at Centre for Health Technologies (CHT), University of Technology, Sydney (UTS). The UTS Human Research Ethics Committee (UTS HREC REF NO. ETH16-0266) approved this study and an informed consent was obtained from all participants before the commencement of data collection. We have 10 volunteers in total with ages from 25 to 47 and height from $168 \mathrm{~cm}$ to $180 \mathrm{~cm}$. The smartphone and sensors have been calibrated prior to the experiments based on the methods introduced in Subsection 2.2 Pre-processing. We also implemented the experiments to test the selected features and the proposed method for gait symmetry analysis.

This experiment can be separated into two parts. In the first part, we tested the validity of the proposed features for post fall analysis by using the data obtained from iPhone 6s. In this part, one iPhone was attached to the upper arm while the other iPhone was placed in the right pocket of volunteer's trouser as shown in Fig.(1). The application 'POWERSENSE' was used to record data from iPhone $6 s$, and the output frequency was set to $100 \mathrm{~Hz}$ for both iPhones.

First, the volunteers were requested to get up from the floor without carrying any sandbags. We collected the data from the first run as the baseline data. In order to simulate the scenario of serious fall, the volunteer carried a set of
$10 \mathrm{~kg}$ sandbags while getting up from the floor. Each volunteer executed both of the tests and 10 set of experiments in total.

For symmetric analysis, the volunteer is asked to wear the IMU modules on both ankles as shown in Fig.(5). The data updating frequency we used was $500 \mathrm{~Hz}$. We applied 3 rd order discrete wavelet transfer (Haar wavelet) on the raw data to remove noise as introduced in pre-processing section. For this experiment, we assumed that the volunteer was hurt seriously on the right side of the body, so we took right foot as the object to be examined. First, the volunteer walked normally without sandbag, and the gyroscope data from z-axis was recorded for 20 steps for symmetric analysis in normal condition. Then, sandbag was added to volunteer's right foot, and the gyroscope data from z-axis for 20 steps are recorded again. Based on these data, we calculated the proposed symmetric index (20) and took the average value for 20 steps.

\subsection{Experiment Results and Discussions}

For the first part of the experiment, we tested the features of getting up from floor by using iPhone $6 \mathrm{~s}$ as the motion sensor. Initially, we use the value from normal getting up without load as baseline. Then, we used this baseline value divide the value from getting up with weight to obtain the percentage of change. Table.(1) and Table.(2) shows the percentage change.

Table 1. Experimental results regarding features of getting up from floor with iPhone on upper arm

\begin{tabular}{lcc}
\hline Feature & Mean value (\%) & Mini-Max (\%) \\
\hline$T_{t d}$ & 147.6 & $110.7-196.0$ \\
\hline$R M S \omega_{x}$ & 87.87 & $51.10-137.6$ \\
$R M S \omega_{y}$ & 113.4 & $54.30-268.4$ \\
$R M S \omega_{z}$ & 108.2 & $69.33-140.8$ \\
\hline$S M_{x}$ & 680.9 & $221.0-1848$ \\
$S M_{y}$ & 671.5 & $165.1-2458$ \\
$S M_{y}$ & 799.3 & $209.4-2481$ \\
\hline$W A\left(a_{x}\right)$ & 281.9 & $159.1-393.1$ \\
\hline$S S C\left(a_{x}\right)$ & 199.5 & $137.0-310.4$ \\
\hline \hline
\end{tabular}

The experimental results indicate, generally, the percentage changes regarding most of the features are quite big. Some features, e.g., Smoothness (SM), Wilson Altitude (WA) and Slope Sign Change (SSC), can have significant changes. It should be pointed out that the calculation of Wilson Altitude and Slope Sign Change are based on the value of world coordinate in $\mathrm{x}$-axis, based on which significant difference can be observed among all axes from the accelerometer and gyroscope. From the experimental results listed in Table 1, it can be expected that a single feature threshold method should be able to reliably discriminate re- 
Table 2. Experimental results regarding features of getting up from floor with iPhone in Pocket

\begin{tabular}{lcc}
\hline Feature & Mean value $(\%)$ & Mini-Max $(\%)$ \\
\hline$T_{t d}$ & 140.1 & $107.7-179.8$ \\
\hline$R M S \omega_{x}$ & 110.4 & $55.01-137.7$ \\
$R M S \omega_{y}$ & 119.5 & $51.91-272.7$ \\
$R M S \omega_{z}$ & 144.1 & $72.80-230.5$ \\
\hline$S M_{x}$ & 472.9 & $131.7-1040$ \\
$S M_{y}$ & 450.3 & $139.8-1088$ \\
$S M_{y}$ & 510.8 & $135.7-1147$ \\
\hline$W A\left(a_{x}\right)$ & 250.5 & $109.5-288.9$ \\
\hline$S S C\left(a_{x}\right)$ & 180.2 & $116.4-261.1$ \\
\hline \hline
\end{tabular}

Table 3. Experimental results regarding symmetric analysis from ImeasureU IMU module

\begin{tabular}{|c|c|c|c|c|c|}
\hline \multirow[t]{2}{*}{ No. } & \multicolumn{2}{|c|}{ Normal Set $(\%)$} & \multicolumn{2}{|c|}{ Sandbag Set $(\%)$} & \multirow[t]{2}{*}{$\mathrm{P}$} \\
\hline & Mean & Min-Max & Mean & Min-Max & \\
\hline 1 & 0.48 & $0.12-1.16$ & 1.22 & $0.93-1.61$ & $2.8 \mathrm{E}-03$ \\
\hline 2 & 0.40 & $0.16-0.96$ & 1.24 & $0.96-1.69$ & $8.2 \mathrm{E}-05$ \\
\hline 3 & 0.48 & $0.15-0.89$ & 1.22 & $0.88-1.70$ & 8.2E-05 \\
\hline 4 & 0.42 & $0.14-0.97$ & 1.30 & $1.07-1.64$ & 4.1E-05 \\
\hline 5 & 0.52 & $0.16-1.32$ & 1.72 & $1.05-3.76$ & 2.9E-04 \\
\hline 6 & 0.87 & $0.24-2.51$ & 1.68 & $0.95-3.71$ & 2.4E-02 \\
\hline 7 & 0.65 & $0.11-1.51$ & 1.77 & $1.16-3.70$ & $1.9 \mathrm{E}-03$ \\
\hline 8 & 0.68 & $0.09-1.60$ & 1.31 & $0.96-1.69$ & $4 \mathrm{E}-03$ \\
\hline 9 & 0.51 & $0.17-0.79$ & 1.37 & $1.12-2.13$ & 4.1E-05 \\
\hline 10 & 0.65 & $0.26-1.58$ & 1.57 & $1.01-3.24$ & $7.8 \mathrm{E}-3$ \\
\hline
\end{tabular}

ally fall from ADL when using the measurement from the upper arm. However, if the iPhone is placed in the pocket (see Table 2), because it is free to move in the pocket when getting up, this free movement greatly degrades the experimental results. In this case, it is necessary to use multiple features and apply pattern classification methods, such as Support Vector Machine (SVM), for improving the classification accuracy. In the next step, we plan to carry out more experiments to significantly train and test a multiple feature-based classifier to improve the classification accuracy for the case that smartphone is placed in the pocket.

For the second part of the experiments, a symmetric analysis is also included to detect the severity of fall. The difference between normal walking and walking with sandbag is significant in terms of the asymmetric index (20) proposed in this paper. Table.(3) recorded the results with $P$ value calculated by Wilcoxon Signed-Rank Test. The results also indicate that DWT can effectively eliminate noise.

Table.(3) shows that the mean asymmetric index $\left(\mathcal{I}_{\text {diff }}\right)$ is quite different, i.e., the mean asymmetric indices are significantly larger when walking with sandbags. Furthermore, the $P$ values based on Wilcoxon Signed-Rank Test are all less than 0.05 , which indicate the difference between with bag and without bag, in term of asymmetric index $\left(\mathcal{I}_{\text {diff }}\right)$, is significant. From SensorTag, we obtained similar results. If more features extracted from individual user's ADL, we believe this proposed symmetric analysis can be applied for the assessment of the severity of fall to facilitate post fall analysis clinically.

\section{Conclusion}

This paper investigates inertial sensor based post fall analysis in order to significantly reduce false alarming of wearable sensor based fall detection. Firstly, several features of getting up from the floor are proposed and tested based on the IMU embedded in the smartphone. It has been demonstrated when the smartphone is attached to the upper arm, by using one of the proposed features (e.g., Smoothness (SM), Wilson Altitude (WA) and Slope Sign Change (SSC)), a simple threshold-based approach can potentially achieve good classification result. This study also indicates that when the smartphone is placed inside the pocket, to improve the classification accuracy, multiple-feature based classification approach should be explored. Furthermore, an asymmetric index is proposed in this study which has the potential to well evaluate the severity of a fall event.

\section{References}

[1] W. H. O. Ageing and L. C. Unit, WHO global report on falls prevention in older age. World Health Organization, 2008.

[2] R. Igual, C. Medrano, and I. Plaza, "Challenges, issues and trends in fall detection systems," Biomed. Eng. Online, vol. 12, no. 66, pp. 1-66, 2013.

[3] N. D. Lane, E. Miluzzo, H. Lu, D. Peebles, T. Choudhury, and A. T. Campbell, "A survey of mobile phone sensing," IEEE Communications Magazine, vol. 48, no. 9, pp. 140-150, 2010.

[4] D. M. Karantonis, M. R. Narayanan, M. Mathie, N. H. Lovell, and B. G. Celler, "Implementation of a realtime human movement classifier using a triaxial accelerometer for ambulatory monitoring," IEEE Transactions on Information Technology in Biomedicine, vol. 10, no. 1, pp. 156-167, 2006.

[5] M. Kangas, I. Vikman, J. Wiklander, P. Lindgren, L. Nyberg, and T. Jämsä, "Sensitivity and specificity of fall detection in people aged 40 years and over," Gait \& posture, vol. 29, no. 4, pp. 571-574, 2009.

[6] A. K. Bourke, P. Van de Ven, M. Gamble, R. O’Connor, K. Murphy, E. Bogan, E. McQuade, 
P. Finucane, G. ÓLaighin, and J. Nelson, "Assessment of waist-worn tri-axial accelerometer based falldetection algorithms using continuous unsupervised activities," in Engineering in Medicine and Biology Society (EMBC), 2010 Annual International Conference of the IEEE. IEEE, 2010, pp. 2782-2785.

[7] M. Yuwono, B. D. Moulton, S. W. Su, B. G. Celler, H. T. Nguyen et al., "Unsupervised machine-learning method for improving the performance of ambulatory fall-detection systems," Biomed Eng Online, vol. 11, no. 9, 2012.

[8] H. Kerdegari, K. Samsudin, A. R. Ramli, and S. Mokaram, "Evaluation of fall detection classification approaches," in Intelligent and Advanced Systems (ICIAS), 2012 4th International Conference on, vol. 1. IEEE, 2012, pp. 131-136.

[9] T. Zhang, J. Wang, L. Xu, and P. Liu, "Fall detection by wearable sensor and one-class SVM algorithm," in Intelligent computing in signal processing and pattern recognition. Springer, 2006, pp. 858-863.

[10] F. Bagalà, C. Becker, A. Cappello, L. Chiari, K. Aminian, J. M. Hausdorff, W. Zijlstra, and J. Klenk, "Evaluation of accelerometer-based fall detection algorithms on real-world falls," PloS one, vol. 7, no. 5, p. e37062, 2012.

[11] L. Schwickert, C. Oberle, C. Becker, U. Lindemann, J. Klenk, M. Schwenk, A. Bourke, and W. Zijlstra, "Model development to study strategies of younger and older adults getting up from the floor," Aging clinical and experimental research, pp. 1-11, 2015.

[12] Q. G. Q. Health. (2016) Queensland stay on your feet. [Online]. Available: https://www.health.qld.gov.au/stayonyourfeet/

[13] J. L. Marins, X. Yun, E. R. Bachmann, R. B. McGhee, and M. J. Zyda, "An extended Kalman filter for quaternion-based orientation estimation using MARG sensors," in Intelligent Robots and Systems, 2001. Proceedings. 2001 IEEE/RSJ International Conference on, vol. 4. IEEE, 2001, pp. 2003-2011.

[14] S. O. Madgwick, A. J. Harrison, and R. Vaidyanathan, "Estimation of IMU and MARG orientation using a gradient descent algorithm," in 2011 IEEE International Conference on Rehabilitation Robotics. IEEE, 2011, pp. 1-7.

[15] I. Frosio, F. Pedersini, and N. A. Borghese, "Autocalibration of triaxial MEMS accelerometers with automatic sensor model selection," Sensors Journal, IEEE, vol. 12, no. 6, pp. 2100-2108, 2012.

[16] L. Ye and S. W. Su, "Optimum experimental design applied to MEMS accelerometer calibration for 9parameter auto-calibration model," in Engineering in
Medicine and Biology Society (EMBC), 2015 37th Annual International Conference of the IEEE. IEEE, 2015, pp. 3145-3148.

[17] M. S. Grewal, L. R. Weill, and A. P. Andrews, Global positioning systems, inertial navigation, and integration. John Wiley \& Sons, 2007.

[18] O. Rioul and P. Duhamel, "Fast algorithms for discrete and continuous wavelet transforms," IEEE Transactions on Information Theory, vol. 38, no. 2, pp. 569-586, 1992.

[19] K. Englehart and B. Hudgins, "A robust, real-time control scheme for multifunction myoelectric control," IEEE Transactions on Biomedical Engineering, vol. 50, no. 7, pp. 848-854, 2003.

[20] D. McGrath, B. R. Greene, K. J. ODonovan, and B. Caulfield, "Gyroscope-based assessment of temporal gait parameters during treadmill walking and running," Sports Engineering, vol. 15, no. 4, pp. 207213, 2012.

[21] B. R. Greene, D. McGrath, R. ONeill, K. J. ODonovan, A. Burns, and B. Caulfield, "An adaptive gyroscope-based algorithm for temporal gait analysis," Medical \& biological engineering \& computing, vol. 48, no. 12, pp. 1251-1260, 2010.

[22] B. Sun, Y. Wang, and J. Banda, "Gait characteristic analysis and identification based on the iPhones accelerometer and gyrometer," Sensors, vol. 14, no. 9, pp. $17037-17054,2014$. 